\title{
Automated extraction of corn leaf points from unorganized terrestrial LiDAR point clouds
}

\author{
Wei Su' ${ }^{1,2}$, Mingzheng Zhang ${ }^{1,2}$, Junming $\mathrm{Liu}^{1,2^{*}}$, Zhongping Sun ${ }^{3}$ \\ (1. College of Land Science and Technology, China Agricultural University, Beijing 100083, China; \\ 2. Key Laboratory of Remote Sensing for Agri-Hazards, Ministry of Agriculture, Beijing 100083, China; \\ 3. Satellite Environment Center, Ministry of Environmental Protection, Beijing 100094, China)
}

\begin{abstract}
Terrestrial LiDAR data can be used to extract accurate structure parameters of corn plant and canopy, such as leaf area, leaf distribution, and 3D model. The first step of these applications is to extract corn leaf points from unorganized LiDAR point clouds. This paper focused on an automated extraction algorithm for identifying the points returning on corn leaf from massive, unorganized LiDAR point clouds. In order to mine the distinct geometry of corn leaves and stalk, the Difference of Normal (DoN) method was proposed to extract corn leaf points. Firstly, the normals of corn leaf surface for all points were estimated on multiple scales. Secondly, the directional ambiguity of the normals was eliminated to obtain the same normal direction for the same leaf distribution. Finally, the DoN was computed and the computed DoN results on the optimal scale were used to extract leave points. The quantitative accuracy assessment showed that the overall accuracy was $94.10 \%$, commission error was $5.89 \%$, and omission error was $18.65 \%$. The results indicate that the proposed method is effective and the corn leaf points can be extracted automatically from massive, unorganized terrestrial LiDAR point clouds using the proposed DoN method.

Keywords: corn leaves, terrestrial LiDAR, cloud points, automatic extraction, crop growth monitoring, phenotyping, difference of normal (DoN), directional ambiguity of the normals

DOI: $10.25165 /$ j.ijabe.20181103.3177
\end{abstract}

Citation: Su W, Zhang M Z, Liu J M. Automated extraction of corn leaf points from unorganized terrestrial LiDAR point clouds. Int J Agric \& Biol Eng, 2018; 11(3): 166-170.

\section{Introduction}

Crop leaves play a significant role for crop plants ${ }^{[1]}$, for example, they determines light interception and plant productivity ${ }^{[2]}$. Therefore, the estimation of leaf structural and biophysical parameters such as leaf area ${ }^{[3]}$, leaf morphology, leaf structure, leaf chlorophyll content etc., is very important for crop growth monitoring ${ }^{[4]}$. Corn (Zea mays) is one of the most widely planted crops $^{[5]}$. And the estimation of these structural and biophysical parameters for corn leaf is very important for crop growth monitoring. Unfortunately, the existence of corn stalk affects the estimation accuracy of these parameters ${ }^{[6]}$. Therefore, the identification of corn leaves is known as one of the important factors for leaf parameters estimation ${ }^{[7,8]}$. Remote sensing technique can obtain these parameters in a large imaging area in a short time ${ }^{[9]}$, which is vital in agricultural application ${ }^{[10-12]}$. Terrestrial LiDAR is an active remote sensing technique ${ }^{[13]}$, which provides highly accurate point clouds (vertical precision and horizontal precision $\leq 2 \mathrm{~mm}$ ) of corn canopy ${ }^{[14]}$. Therefore, the LiDAR point clouds can be used to extract accurate leaf structure parameters non-destructively ${ }^{[15]}$. The primary work for this application is to extract leaf points ${ }^{[16]}$.

\section{Received date: 2017-01-08 Accepted date: 2018-01-29}

Biographies: Wei Su, PhD, Associate Professor, research interests: crop growth monitoring by combining LiDAR and satellite remote sensing images, Email: suwei@cau.edu.cn; Mingzheng Zhang, PhD candidate, research interests: crop growth monitoring by combining LiDAR and satellite remote sensing images, Email: wuyuezhiye@yeah.net; Zhongping Sun, PhD, Professor, research interests: application of environmental remote sensing, Email: sunnybnu114@163.com.

*Corresponding author: Junming Liu, PhD, Associate Professor, research interests: low temperature disaster monitoring using remote sensing technique. College of Land Science and Technology, China Agricultural University, Beijing 100083, China. Tel: +86-10-62737855, Email: liujunming2000@163.com.
The raw terrestrial LiDAR points are massive, unorganized $^{[17,18]}$. Therefore, the extraction of corn leaf points from terrestrial LiDAR points is a difficult but crucial work in agricultural application $^{[19]}$. The difficulties also arise from the inherent complexity of point clouds within the corn canopy ${ }^{[20]}$. The existing extraction approaches can be basically classified as three groups: edge-based ${ }^{[21,22]}$, region-based ${ }^{[23,24]}$, and hybrid ${ }^{[25,26]}$ algorithms. The edge-based algorithms detect the difference between leaves and stalk using the features such as the normal vector, gradients, and principal curvatures in a cross-section. The points are usually classified according to the differences of features between adjacent points. The region-based methods classify the data by detecting continuous surfaces that have homogeneity or similar geometrical properties ${ }^{[14]}$. Region-based methods are more robust (less sensitive to noise) than edge-based methods. However, there are over- or under-segmentation results and the border ambiguous ${ }^{[27]}$. The hybrid methods use a combination of edge- and region-based analysis techniques, which analyzes points using several kinds of geometrical, image-metrical, or even texture-metrical features.

In view of the massive, unorganized characteristic of terrestrial LiDAR point clouds, this paper proposes a multi-scale DoN operator for the extraction of corn leaf points ${ }^{[19]}$. The main contribution of this study is to propose the DoN method to extract corn leaf points automatically, whose geometrical surface is thin, irregular, and curly.

This study is aiming at extracting the corn leaves LiDAR points from massive corn plants points. The estimated multi-scale DoNs are used to identify leaves points based on the obvious difference of normals for corn leaves points and corn stalk points. The experimental results indicate that the proposed DoN method is effective and the corn leaf points can be extracted automatically 
from massive, unorganized terrestrial LiDAR point clouds with the high overall accuracy and low commission error and omission error.

\section{Data source and pre-processing}

\subsection{Data source}

The study area is located in a greenhouse of China Agricultural University, Beijing, China. Several corn plants were scanned in this greenhouse on December $4^{\text {th }}, 2011$, using a FARO Focus3D laser scanner (http://www.faro.com/products/3d-surveying/laserscanner-faro-focus-3d/overview). The corn was in the jointing stage, and the corn plant was around $1.9 \mathrm{~m}$ tall. There were 11 corn plants in scanned area. We selected two plants to extract corn leaf points in this study. Table 1 shows the laser scanning parameters. The attributes of LiDAR points include $x, y$, and $z$ coordinates, the intensity etc. There were two laser scanning stations for acquiring complete points of corn plants. One station is in the northwest of greenhouse and the other is in the southwest of greenhouse, and the distance between them are $12 \mathrm{~m}$. Figure 1 shows the sketch of laser scanning for corn plant, and the laser lights illuminated from two stations and reached on corn leaves (red dots) and corn stalk (green triangles), then the returned laser lights are received by laser receiver. Every point pi $(i=1,2, \ldots, n)$ is represented by $(X i, Y i, Z i)^{T}$ in the local coordinate system $(L C S)$. Therefore, they have different local coordinate system for the points returned from these two stations. These two different local coordinate systems were uniformed in the following data pre-processing stage.

Table 1 3D laser scanning parameters of FARO Focus3D (maize scene) scanners for corn plant scene

\begin{tabular}{lc}
\hline \multicolumn{1}{c}{ Parameters } & Values \\
\hline Scanning speed & $48.8 \mathrm{mrad} / \mathrm{s}$ \\
Scanning angle & $360^{\circ}$ (horizontal), $305^{\circ}$ (vertical) \\
Precision (systemic range error) & $\leq 2 \mathrm{~mm}$ (within $25 \mathrm{~m}$ ) \\
Distance accuracy & $8 \mathrm{~mm}$ (within $50 \mathrm{me}$, single point scanning) \\
Angular resolution & 1.8 arcsec (horizontal), 1.8 arcsec (vertical) \\
Vertical scanning step & $0.009^{\circ}\left(360^{\circ}\right)$ \\
Horizontal scanning step & $0.009^{\circ}\left(360^{\circ}\right)$ \\
Wavelength & $905 \mathrm{~nm}$ \\
\hline
\end{tabular}

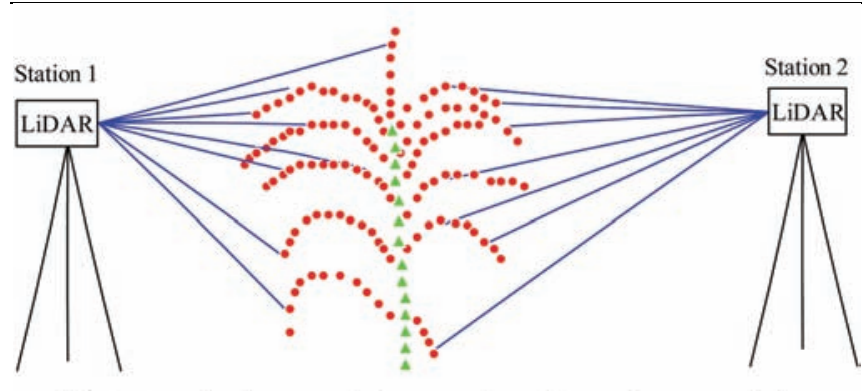

- Points returned on leaves A Points returned on stalk - Laser scanning beam

Figure 1 Sketch of laser scanning for corn plant

\subsection{Data pre-processing}

There were some isolated, invalid points returned on the wall, the roof and the lamp of greenhouse. Therefore, the first step was removing these invalid points from the corn plant points. An enveloped cuboid was used to identify the points returned on corn plants, thus the points returned on the wall, the roof and the lamp of greenhouse have been removed. Figure 2a shows the raw corn plant points from two scanning stations, we can see that there was obvious geometric shift between the points of two stations. Therefore, the points were registered using tie-points. The algorithm for LiDAR points registration were was developed using the $\mathrm{C}++$ programming language in this study. The principle of LiDAR points registration is to rotate the coordinates and make the rigid transformation for the LiDAR points scanned from two stations. And the transformation matrix was built for registration. For the LiDAR points $p_{\mathrm{i}}$ and $q_{\mathrm{i}}$ from two stations, the optimal rotation matrix $\boldsymbol{R}$ and translation matrix $\mathrm{T}$ are built for LiDAR points registration and the value of function $\mathrm{f}(\mathrm{R}, \mathrm{T})=\sum_{1}^{n}\left\|p_{i}-\left(R q_{i}+T\right)\right\|^{2}$ is minimized. The coordinate transformation matrix $\boldsymbol{T}$ is defined as follows:

$$
\left[\begin{array}{l}
X \\
Y \\
Z
\end{array}\right]=\mu R(\alpha, \beta, \gamma)\left[\begin{array}{l}
x \\
y \\
z
\end{array}\right]+T
$$

where, $\mu$ is a coefficient that counting the scale difference between these two coordinate systems; $\alpha, \beta, \gamma$ are rotation coefficients; $\boldsymbol{T}$ is translation matrix:

$$
T=\left[\begin{array}{l}
x_{0} \\
y_{0} \\
z_{0}
\end{array}\right]
$$

These seven conversion coefficients are needed to work out for registering the LiDAR points from two stations.

Using our registration code, we unify the positive plane and the reference plane to match the LiDAR points from two stations. The matched points are used to extract corn leaf points. There are 89940 points after registration (Figure 2b), including the points returned on corn plants and ground. Finally, we classified the points into ground points and corn plants points based on the height. Figure $2 \mathrm{c}$ shows the classified points returned on two corn plants.

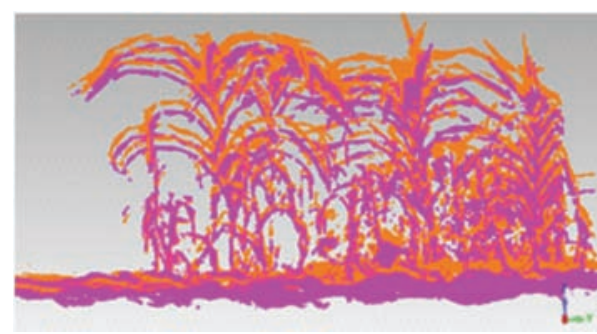

a. Scanned point clouds from two stations

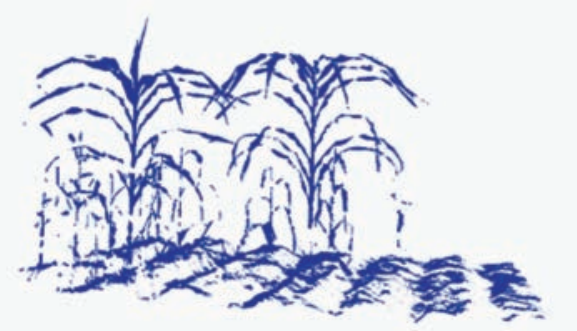

b. Point clouds after registration

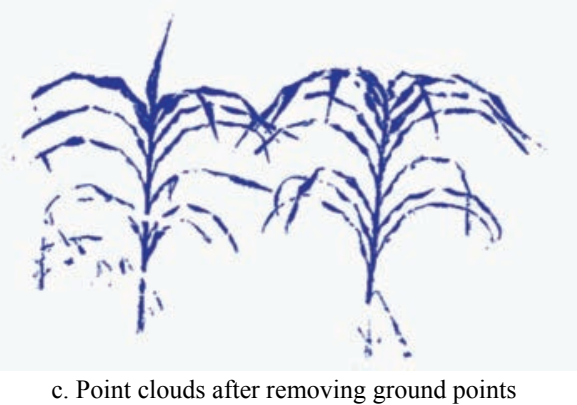

Figure 2 LiDAR points for (a) corn plants obtained from two scanning positions, (b) the same points after registration, and (c) the points after removing ground points 


\section{Method}

The proposed method aims to extract corn leaf points from massive LiDAR points using the difference of the normal vectors calculated at two scales. The flowchart is as Figure 3. As shown in Figure 3, there are three vital steps for this extraction: 1) estimating the normals of corn plants using the principal components of neighborhood; 2) eliminating the directional ambiguity of normals using undirected graph traversal method; 3 ) computing the difference of normal. The DoN is used to extract corn leaf points.

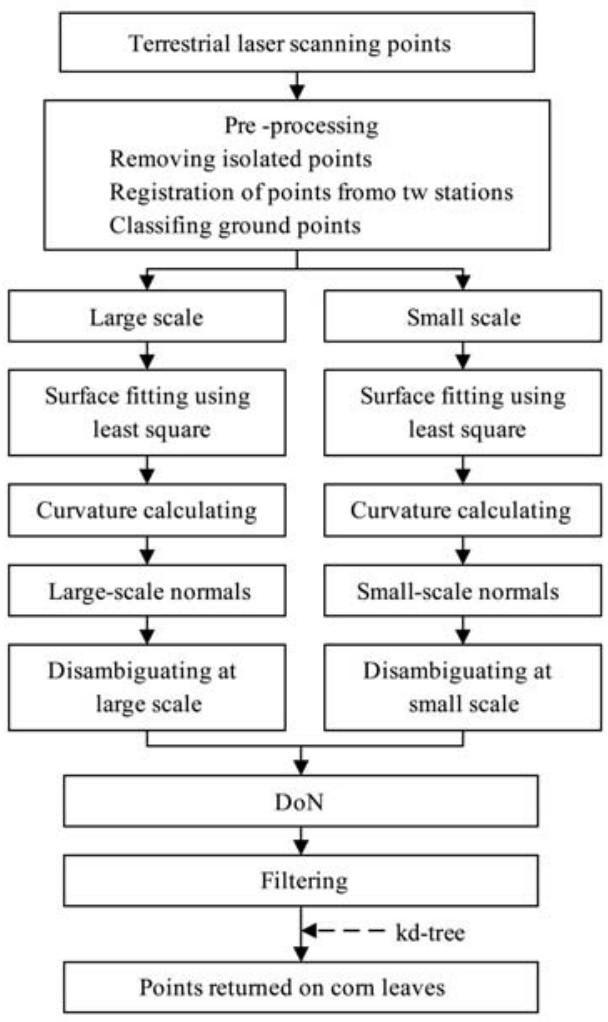

Figure 3 Flowchart of extracting corn leaf points with DoN method

\subsection{Estimating the normals of corn leaf points}

The massive unorganized LiDAR points are stored in kd-tree format. For each point, a least squares local plane is fitted with its $k$ nearest neighbors ${ }^{[17]}$. Tangent plane $S$ is a discrete point set in $k$-nearest neighbor, which is computed as:

$$
S(n, d)=\arg \min \sum_{i=1}^{k}\left(n p_{i}-d\right)^{2}
$$

where, $n$ is the normal vector; $p_{i}$ is point cloud; $d$ is the distance between point $p_{i}$ and the origin of coordinates.

The centroid of tangent plane $S$ is $\bar{p}_{i}$, which is computed as:

$$
\bar{p}_{i}=\frac{1}{k} \sum_{j=0}^{k-1} p_{i, j}
$$

The normal vectors was estimated by finding the tangent plane $(M)$ using the principal components of a local neighborhood for each point. The solution for estimating the surface normal is the eigenvectors and eigenvalues using principal-components analysis (PCA) of a covariance matrix created from the nearest neighbors of the studied point. The covariance matrix $(M)$ is computed as:

$$
M=\frac{1}{k} \sum_{i=1}^{k}\left(p_{i}-\bar{p}\right)\left(p_{i}-\bar{p}\right)^{T}
$$

where, $\left(p_{i}-\bar{p}\right)$ is a column vector; $\left(p_{i}-\bar{p}\right)^{T}$ is transposed column vector; $\bar{p}$ is the centroid of neighboring points.

\subsection{Eliminating the directional ambiguity of normals}

The directional ambiguity is within the surface normals: there are two equal but opposite (negative and positive) normals for any object surface, both of them are mathematically valid. In many applications, this ambiguity can be resolved using the view point because the correct normal is always the one in the hemisphere that points towards the view point. Disambiguation of the normals is done by negating one of the normals if $n\left(p, r_{1}\right)-n\left(p, r_{2}\right)>\pi / 2$, i. e. the angle between the two normals with different searching radius is greater than $90^{\circ}$. This assumes that the correct normals must be within an angle of $\pi / 2$ for corn leaves points.

\subsection{Computing DoN}

Normals estimated with a specific support radius share some similarities with a scale-space operator ${ }^{[19]}$. If the structure of the larger neighborhood differs significantly from that of a smaller neighborhood, the directions of the two estimated normals are likely to be different obviously. Thus the difference of normals between these two radii for corn leaves points and corn stalk points is significant, which can be used to identify corn leaf points. The $\operatorname{DoN}(\Delta)$ for a point cloud is defined as follows:

$$
\Delta n\left(p, r_{1}, r_{2}\right)=\left[n\left(p, r_{1}\right)-n\left(p, r_{2}\right)\right] / 2
$$

where, $r_{1}<r_{2}, r_{1}$ and $r_{2}$ are two searching radii. $\Delta n\left(p, r_{1}, r_{2}\right)$ is the difference of normals in two scales, the attributes of which include the $x, y$, and $z$ coordinates, normals and normals' curvature.

\section{Results and analysis}

\subsection{Estimated normals and DoN features for corn plants}

The selection of optimum scale for normal estimation is vital for corn leaves points identification, the principle of which is maximizing the magnitude of the DoN for the points set within leaves points and minimizing the DoN value between leaves points and stalk points. Ioannou et al. ${ }^{[19]}$ found that they got the best extraction result when the $\Delta n(p)$ vectors is computed on two scales whose ratio $\left(r_{2} / r_{1}\right)$ is 10 , for an example, $r_{2}=2 \mathrm{~m}$ and $r_{1}=0.2 \mathrm{~m}$. Therefore, we computed the normal on three groups of scales for corn leaf points extraction, $(0.1 \mathrm{~m}, 1 \mathrm{~m}),(0.2 \mathrm{~m}, 2 \mathrm{~m})$, $(0.4 \mathrm{~m}, 4 \mathrm{~m})$, which is as Figure 4 . The black points are LiDAR points and the white lines are normals of points in Figure 4. And Figure 4a and Figure 4b, Figure $4 \mathrm{c}$ and Figure 4d, Figure $4 \mathrm{e}$ and Figure $4 \mathrm{f}$ are the calculated normals using searching radius of $(0.1 \mathrm{~m}, 1 \mathrm{~m}), \quad(0.2 \mathrm{~m}, 2 \mathrm{~m})$, and $(0.4 \mathrm{~m}, 4 \mathrm{~m})$ respectively. We can see that there is different vector direction, vector magnitude for the three pairs of normal results. Especially for Figure $4 \mathrm{a}$ and Figure $4 \mathrm{~b}$, there are obvious different normal vector using different scales.

The DoN values for every LiDAR points, $\Delta n\left(p, r_{1}, r_{2}\right)$, are computed using $(0.1 \mathrm{~m}, 1 \mathrm{~m}),(0.2 \mathrm{~m}, 2 \mathrm{~m})$, and $(0.4 \mathrm{~m}, 4 \mathrm{~m})$. Figure 5 is the resulted DoN vectors $(|\Delta n(p)|)$ at scales of 0.1 and $1 \mathrm{~m}, 0.2$ and $2 \mathrm{~m}, 0.4$ and $4 \mathrm{~m}$ respectively. Figure $5 \mathrm{a}$ is the pre-processed LiDAR points returned on two corn plants, and Figure 5b, Figure 5(c), Figure $5 \mathrm{~d}$ are the resulted DoN vectors at such three scales as $(0.1 \mathrm{~m}, 1 \mathrm{~m}),(0.2 \mathrm{~m}, 2 \mathrm{~m}),(0.4 \mathrm{~m}, 4 \mathrm{~m})$ respectively. From these three groups of DoN vector results, we can see that the DoN result at $(0.1 \mathrm{~m}, 1 \mathrm{~m})$ showed the strongest difference between corn leaves points and corn stalk points. Therefore, the $(0.1 \mathrm{~m}, 1 \mathrm{~m})$ is the optimal scale for corn leaf points extraction in our analysis. 


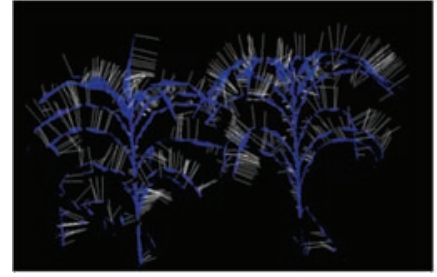

a. $r=0.1 \mathrm{~m}$

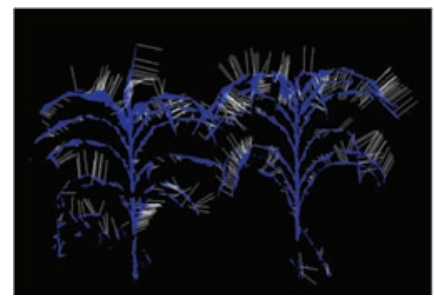

c. $r=0.2 \mathrm{~m}$

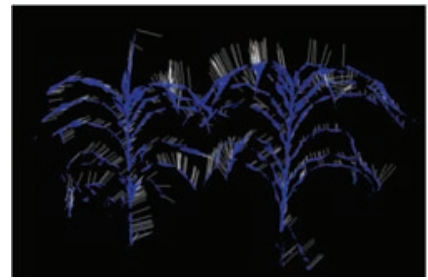

e. $r=0.4 \mathrm{~m}$



b. $r=1 \mathrm{~m}$

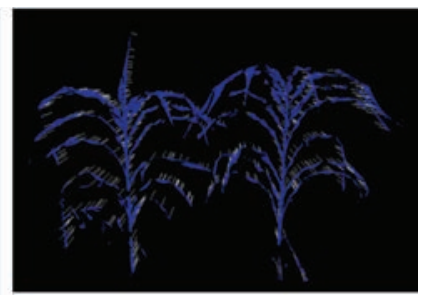

d. $r=2 \mathrm{~m}$

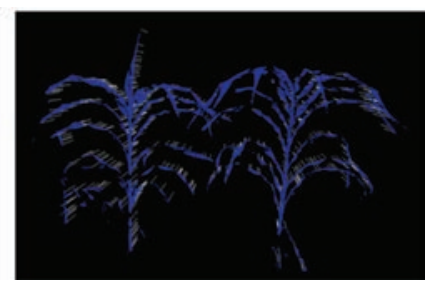

f. $r=4 \mathrm{~m}$
Figure 4 Different normals of corn plant points using

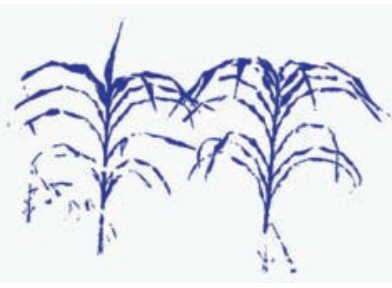

a. Pre-processed point cloud

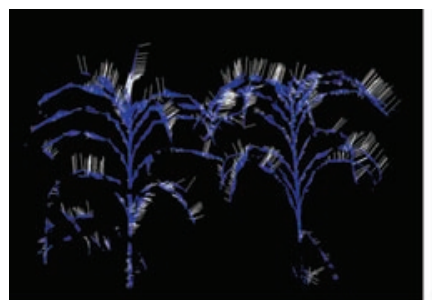

c. $|\Delta n(0.2 \mathrm{~m}, 2 \mathrm{~m})|$

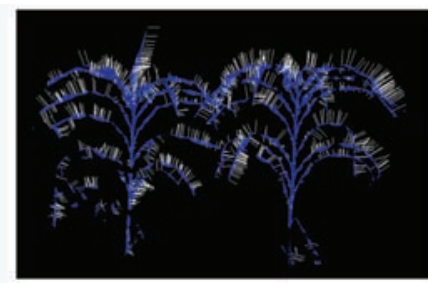

b. $|\Delta n(0.1 \mathrm{~m}, 1 \mathrm{~m})|$



d. $|\Delta n(0.4 \mathrm{~m}, 4 \mathrm{~m})|$
Figure 5 Computed DoN for corn plants using scales

\subsection{Filtered DoN results at multi-scales}

The multi-scale DoN operators are used to identify corn leaves points and corn stalk points. Figure 6 shows the DoN filtered results for the corn plants. At the smallest scale of $(0.1 \mathrm{~m}, 1.0 \mathrm{~m})$, shown in Figure $6 \mathrm{~b}$, the leaves and stalks of the corn are filtered obviously, and the details of corn leaves are complete. Using larger scales of $(0.2 \mathrm{~m}, 2 \mathrm{~m})$ and $(0.4 \mathrm{~m}, 4 \mathrm{~m})$, as shown in Figures $6 \mathrm{c}$ and Figures $6 \mathrm{~d}$, many leave points are missed because these points are classified as stalk using the normal vectors computed on these two scales. On the larger scales, only large objects are preserved, and smaller objects are increasingly removed. Figure 7 is the sketch of incorrect extracted points when the DoN of leave points is the same to the DoN of stalk points. We can see that there are some points returned on top erect leaves (labeled "leave points" in the top of Figure 7) and the lower hanging down leaves (labeled "leave points" in the bottom of Figure 7) are classified as stalk because their normal vector is similar to the normal of corn stalk.

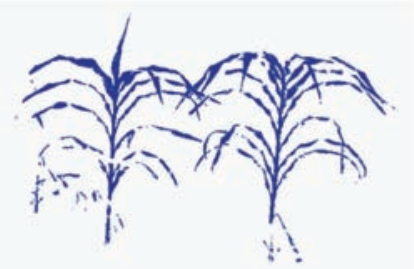

a. Original (91729 points)



c. $|\Delta n(0.2 \mathrm{~m}, 2 \mathrm{~m})| \geq 0.15$

(45373 points)

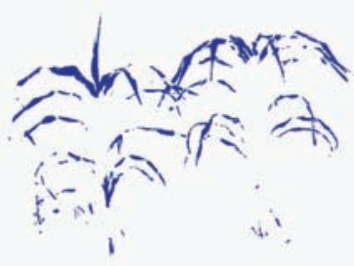

b. $|\Delta n(0.1 \mathrm{~m}, 1 \mathrm{~m})| \geq 0.15$ (57628 points)

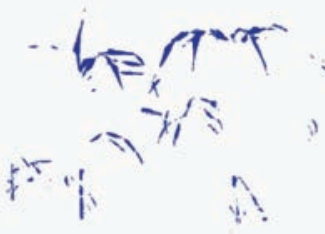

d. $|\Delta n(0.4 \mathrm{~m}, 4 \mathrm{~m})| \geq 0.15$ (32157 points)
Figure 6 Extracted corn leaves points with three scales

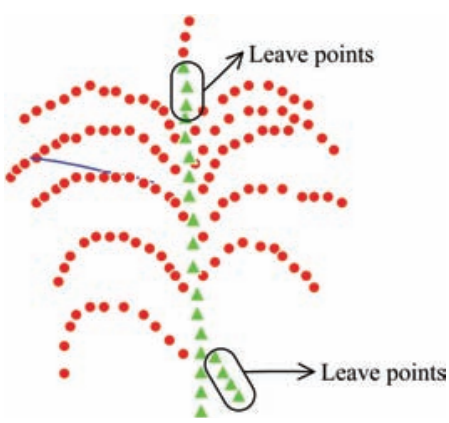

Figure 7 Sketch of incorrect extracted points when the normal of leave points is the same to the normal of corn stalk.

\subsection{Extracted corn leaf points}

After the comparison of extracted corn leaves points on three scales, the scale $(0.1 \mathrm{~m}, 1 \mathrm{~m})$ was used to identify corn leaves points. And a clustering algorithm of a simple Euclidean distance threshold ${ }^{[28]}$ was used to extract corn leaves points and corn stalk points respectively. The parameters of clustering are as follow: distance tolerance is $\mathrm{r} 3$, the minimum distance is 100 cluster points, and the maximum distance is 100000 cluster points. Figure $8 \mathrm{a}$ and Figure $8 \mathrm{~b}$ show the details of corn leaf cluster and corn stalk cluster. It can be seen that the corn leave points can be extracted correctly from corn stalk points.

The accuracy was assessed quantitatively to validate the DoN algorithm for extracting corn leaf points. The leaves points were classified manually from the stalk points, which were the reference points for accuracy assessment. Overall accuracy, commission error, omission error, were used in accuracy assessment. There were 89940 points totally returned on two corn plants. And the overall accuracy was $94.10 \%$, commission error was $5.89 \%$, and omission error was $18.65 \%$ in this study.

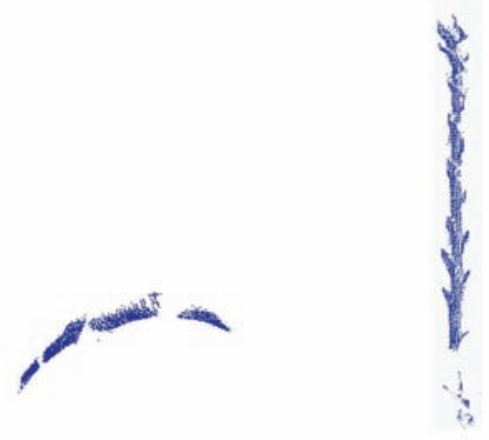

a. Corn leaf cluster

b. Corn stalk cluster

Figure 8 Extracted results of corn leaves points and stalk points 


\section{Discussion}

The DoN operator was used to extract the corn leaves points from unorganized corn plants LiDAR points automatically in this study. The extracted results and accuracy assessment indicated that the extraction was effective. In order to mine the distinct geometry of corn leaves and stalk, the difference of surface normal at different scales was used to identify corn leave points. One important issue for this extraction is finding the optimal search radius, i. e. these two scales ("large scale" and "small scale"). Referencing to the conclusion of Ioannou et al. ${ }^{[19]}$, the ratio of large scale to small scale should be 10 , thus such 3 groups of search radius as $(0.1 \mathrm{~m}, 1 \mathrm{~m}),(0.2 \mathrm{~m}, 2 \mathrm{~m})$, and $(0.4 \mathrm{~m}, 4 \mathrm{~m})$ were chosen for corn leaves points extraction. The computed DoN results (Figure 5) indicated that the $(0.1,1 \mathrm{~m})$ was the optimum one for corn leaf points extraction in our analysis. Thus the $(0.1 \mathrm{~m}, 1 \mathrm{~m})$ search radius was used in the study. The accuracy assessment results showed that the extraction was successful, and the overall accuracy was $94.10 \%$. The disadvantage of this method is that the points returned on top erect leaves and lower drooping leaves cannot be extracted successfully (Figure 7). For this reason, the omission error is relatively high, which reached $18.65 \%$ in this study.

\section{Conclusions}

Compared with the extraction of points returned on buildings, man-made models etc., the corn leaf points extraction is difficult because the corn leaves are thin and curled. This study is aiming at extracting corn leaf points from unorganized LiDAR point clouds automatically. The extracted results indicate that the corn leaf points can be extracted effectively using the DoN method. This study on corn leaf points extraction from massive, unorganized data, is meaningful for agriculture application, especially in phenotyping of corn plants and corn canopy. Further research needs to be done through putting field phenotyping of corn canopy in the future, such as estimating leaf area index, leaf distribution function, biomass, the relationship between these phenotypic parameters and yield estimation etc.

\section{Acknowledgments}

This research was supported by National Natural Science Foundation of Chinar for the project of Growth process monitoring of corn by combining time series spectral remote sensing images and terrestrial laser scanning data (41671433), Dynamic calibration of exterior orientations for vehicle laser scanner based structure features (41371434) and Estimating the leaf area index of corn in whole growth period using terrestrial LiDAR data (41371327).

\section{[References]}

[1] Han W T, Sun Y, Xu T F, Chen, X W, Su K O. Detecting maize leaf water status by using digital RGB images. International Journal of Agricultural \& Biological Engineering, 2014; 7(1): 45-53.

[2] Weraduwagel S M, Chen J, Anozie F C, Morales A, Weise S E, Sharkey T D. The relationship between leaf area growth and biomass accumulation in Arabidopsis thaliana. Frontiers in Plant Science, 2015; 6: 1-21.

[3] Liu Z H, Huang R G, Hu Y M, Fan S D, Feng P H. Generating high spatiotemporal resolution LAI based on MODIS/GF-1 data and combined Kriging-Cressman interpolation. Int J Agric \& Biol Eng, 2016; 9(5): $120-131$.

[4] Huang J X, Sedano F, Huang Y B, Ma H Y, Li X L, Liang S L, et al. Assimilating a synthetic Kalman filter leaf area index series into the WOFOST model to improve regional winter wheat yield estimation. Agricultural \& Forest Meteorology, 2016; 216: 188-202.

[5] Zia S, Sophrer K, Du W Y, Spreer W, Romano G, He X K, Joachim M. Monitoring physiological responses to water stress in two maize varieties by infrared thermography. Int J Agric \& Biol Eng, 2011; 4(3): 7-15.

[6] Kahriman F, Demirel K, Inalpulat M, Egesel C O, Genc L. Using leaf based hyperspectral models for monitoring biochemical constituents and plant phenotyping in maize. Journal of Agricultural Science and Technology, 2016; 18: 1705-1718.

[7] Muller-Linow M, Pinto-Espinosa F, Scharr H, Rascher U. The leaf angle distribution of natural plant populations: assessing the canopy with a novel software tool. Plant Methods, 2015; 11: 1-16.

[8] Yang W, Duan L, Chen G, Xiong L, Liu Q. Plant phenomics and high-throughput phenotyping: accelerating rice functional genomics using multidisciplinary technologies. Current Opinion in Plant Biology, 2013; 16(2): 180-187.

[9] Lin Y, Hyyppä J. A comprehensive but efficient framework of proposing and validating feature parameters from airborne LiDAR data for tree species classification. International Journal of Applied Earth Observation \& Geoinformation, 2016; 46: 45-55.

[10] Huang J X, Sedano F, Huang Y B, Ma H Y, Li X L, Liang S L, et al. Assimilating a synthetic Kalman filter leaf area index series into the WOFOST model to improve regional winter wheat yield estimation. Agricultural \& Forest Meteorology, 2016; 216: 188-202.

[11] Huang J X, Tian L Y, Liang S L, Ma H Y, Becker-Reshef I, Su W, et al. Improving winter wheat yield estimation by assimilation of the leaf area index from Landsat TM and MODIS data into the WOFOST model. Agricultural \& Forest Meteorology, 2015; 204: 106-121.

[12] Huang J X, Ma H Y, Su W, Zhang X D, Huang Y B, Fan J L, et al. Jointly assimilating MODIS LAI and ET products into the SWAP model for winter wheat yield estimation. IEEE Journal of Selected Topics in Applied Earth Observations \& Remote Sensing, 2015; 8(8): 4060-4071.

[13] Buckley S J, Kurz T H, Howell J A, Schneider D. Terrestrial LiDAR and hyperspectral data fusion products for geological outcrop analysis. Computers \& Geosciences, 2013; 54(4): 249-258.

[14] Zheng G, Moskal L M. Leaf orientation retrieval from terrestrial laser scanning (TLS) data. IEEE Transactions on Geoscience and Remote Sensing, 2012; 50: 3970-3979.

[15] Lin Y. LiDAR: An important tool for next-generation phenotyping technology of high potential for plant phenomics? Computers \& Electronics in Agriculture, 2015; 119: 61-73.

[16] Hosoi F, Nakai Y, Omasa K. 3-D voxel-based solid modeling of abroad-leaved tree for accurate volume estimation using portable scanning lidar. ISPRS Journal of Photogrammetry \& Remote Sensing, 2013; 82: 41-48.

[17] Li B, Schnabelr R, Klein R, Cheng Z Q, Dang G, Jin S Y. Robust normal estimation for point clouds with sharp features. Computers \& Graphics, 2010; 34: 94-106.

[18] Lin Y, Herold M. Tree species classification based on explicit tree structure feature parameters derived from static terrestrial laser scanning data. Agricultural \& Forest Meteorology, 2016; 216: 105-114.

[19] Ioannou Y, Taati B, Harrap R, Greenpan M. Difference of normals as a multi-scale operator in unorganized point clouds. Second International Conference on 3D Imaging, Modeling, Processing, Visualization and Transmission (3DIMPVT), Zurich, 2012; pp.501-508.

[20] Das A, Servos J, Waslander S L. Scan registration using segmented region growing NDT. The International Journal of Robotics Research 2014; 33: 1645-1663.

[21] Wani M A, Batchelor B G. Edge-region-based segmentation of range images. IEEE Transaction on Pattern Analysis and Machine Intelligence, 1994; 16: 314-319.

[22] Huang J B, Meng C H. Automatic data segmentation for geometric feature extraction from unorganized 3-D coordinate points. IEEE Transaction on Robotics and Automation, 2001; 17: 268-279.

[23] Besl P J, Jain R C. Segmentation through variable-order surface fitting. IEEE Transaction on Pattern Analysis and Machine Intelligence, 1988; 10: 167-192.

[24] Jiang X Y, Bunke H, Meier U. High-level feature based range image segmentation. Image and Vision Computing, 2000; 18: 817-822.

[25] Köster K, Spann M M. MIR: An approach to robust clustering-application to range image segmentation. IEEE Transaction on Pattern Analysis and Machine Intelligence, 2000; 22: 430-444.

[26] Lavoué G, Dupont F, Baskurt A. A new CAD mesh segmentation method based on curvature tensor analysis. Computer-Aided Design, 2005; 37 975-987.

[27] Nurunnabi A, Belton D, West G. Robust segmentation for multiple plana surface extraction in laser scanning 3D point cloud data. 21st International Conference on Pattern Recognition (ICPR), Tsukuba, 2012; pp.1367-1370.

[28] Rusu R B. Semantic 3D Object Maps for Everyday Manipulation in Human Living Environments, PHD thesis, Computer Science department, Technische Universitaet Muenchen, Germany, October 2009. 